\title{
Differences of the Luminous Principle between Laser devices and LED devices
}

\author{
Li Xincheng ${ }^{1, *}$ \\ ${ }^{1}$ Bur Oak Secondary School, Markham, Ontario, L6E 1G4, Canada
}

\begin{abstract}
The concept of luminescence has been a fascinating concept to mankind. The goal of this paper is to provide insights into the mysterious phenomenon of photoluminescence occurring from natural crystal lattice materials through electron radiation processes. The research methods used in the study involve the detection, identification, and interpretation of the structure, the working principle of the luminescence concept, and the application of the concept of luminescence from written materials. The methods extracted from the materials involve biological and biochemical lab methods based on the existence of phosphorescence, chemiluminescence, bioluminescence, and finally fluorescence. The study paper also highlights the differences between LEDs and LASERS, which are the main types of luminescence producing semiconductor devices. Their variations are presented in aspects of their working principle, the type of luminescence they produce, the respective output power, and the speed, transmitting distance \& cost of each. Photoluminescence is a concept whose applications are widespread in the physical and chemical processes of our daily activities. Therefore, it is important to have a sufficient understanding of the successful applications of luminescent enhancement.
\end{abstract}

\section{Introduction}

The principle of luminescence occurs in the thirdgeneration semiconductor materials, for which photoelectric applications are required. But the third generation of semiconductor materials used in different photoelectric instruments, luminous intensity form path are different. In this paper, the general luminescence principle of the third-generation semiconductor and its application in different optical instruments are suggested. The factors that lead to different luminescence intensity paths or forms was discussed through studies on luminescence principles. The methods that can be used to effectively improve luminous emission efficiency are very significant in applying the third-generation semiconductor[7]. Through this research, scientists in the future design can improve the luminous efficiency of photoelectric instruments related products, energy saving, and environmental protection.

\section{Principles of Semiconductor Lumi- nescence}

The luminous principle can be collectively referred to as the luminescence, which is emitting light or photo responsiveness of such materials without any influence from heat or "cold light". Some of the light-emitting devices which operate under this principle include lightemitting diodes (LEDs) and photo-responsive devices such as solar cells and photodetectors. These devices emit light through the optoelectronic process, in which electrical energy is converted to optical energy in semiconductors [1].

\subsection{Photon release during luminous}

The creation of photoemission involves the excitement of an electron from the ground state (E1). During the transition of the electron from the excited state (E2) to the ground is a double state system, in which a photon is released as shown in figure 1 below.

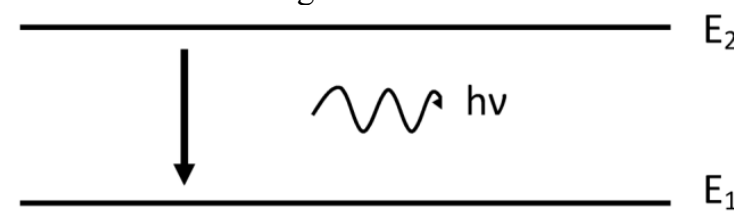

\section{$\mathrm{E}_{1}$}

$\mathrm{E}_{2}$

\subsection{Photoelectric converting principle}

The ground state of semiconductors is referred to the electrons in the valence band whereas the electrons in the excited state are called the conduction band. Therefore, this creates a minimum amount of energy which is required to excite an electron from the ground to the excited state. In the luminescence of semiconductor materials, electrons are similarly excited to higher states like the conduction band and subsequently decay to the ground state. However, the excitement of these semiconductors van is of several types depending on the type of luminescence, for instance, photoluminescence, electro-

*vivian.wang@cas-harbour.org 
luminescence, among others. However, in our case, we are more concerned with LEDs and Lasers, which fall under the electro-luminescence devices. They involve the conversion of electrical energy into light emission, hence producing light.

\section{Electrical process During Semicon- ductor Luminescence}

The Electroluminescence is a product of the active recombination of electrons and holes in a semiconductor material. The excited electrons discharge their energy in form of photons (light). Before the recombination process, the electrons and holes may have been separated by either doping the semiconductor material to create a $p-n$ junction(in semiconductor electroluminescent devices such asLEDs) or else by excitation in which an impact of electrons at great energy is enhanced by a robust electric field, for instance, in electroluminescent displays[2].

The process is therefore is an instantaneous optical process and also an electrical process. All these occur in the $\mathrm{p}-\mathrm{n}$ junction of the semiconductor, where the exciting electric current generates electron-hole pairs. The subsequent recombination of electron-hole pairs, therefore, results in the emission of light. The quantum efficiency of these electroluminescence materials can however be very high indirect bandgap semiconductors having low crystal defects density. Averagely, a single photon is obtained for every electron-hole pair or every electron excited into the device. Therefore, this produces photon energy and the color of the emitted light, all of which are determinants of the bandgap energy of the semiconductor material.[3]

Under the forward voltage, the electron is injected into the $\mathrm{P}$ region from the $\mathrm{N}$ region, and the hole is injected into the $\mathrm{N}$ region from the $\mathrm{P}$ region. Part into the other area of minority carrier with majority carrier compound and shine, assume that light is in the $\mathrm{P}$ region, then injected electrons and valence band holes directly compound and shine, suppose that light is in the $\mathrm{P}$ region, then injected electrons and the valence band hole composite and shine now, or captured by luminescence center first, again with composite hole luminescence. Luminous compounds, which release little energy at a time, cannot form visible light. The higher the luminous composite ratio to the non-luminous composite, the higher the optical quantum efficiency is[6].

The injection process of electrons and holes involves three main stages: transportation, thermal relaxation, and recombination. For transportation, electrons and holes take energy from the semiconductor as they proceed from the metal interaction to the active region. In thermal relaxation, the injected electrons (holes) settle at the foot of the conduction band in the active region. The recombination process involves the creation of an electron-hole pair, either radially or non-radially. The diagram below summarizes how electrons and holes are added into the active region to emit light.

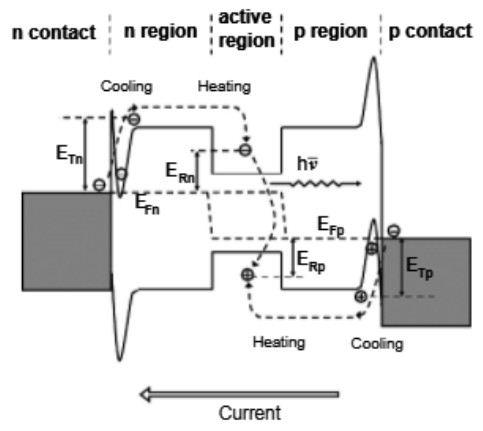

Figure2 Photovoltaic conversion by holes and electrons

Figure 2 shows the schematic band illustration of a semiconductor heterostructure LED when it is forward biased. In this case, both the carrier transport and the process of creating an electron-hole pair are related to electroluminescence, and the special difference of electron-hole typical transportation energies ETn (ETp) and the typical recombination energy ERn (ERp) are also under isothermal settings. The isothermal conditions are achieved by the carrier transport, which offers cooling by adding cladding layers, from the $\mathrm{n}$ and $\mathrm{p}$ contacts to the edges of the active region.

\section{Working Mechanism of Lumines- cence}

The Luminous principle has been of great application in our current lighting systems. Engineers have gone to the extent of developing electroluminescent materials which can stretch more than six times their original length while still emitting light. These are brilliant applications of the optical phenomenon and the electrical phenomenon on which these semiconductor materials emit light in response to the exciting current or strong exciting electric field.

The emission of visible light is usually in the range of approximately 690 nanometers and 400 nanometers. However, the emission of light requires excitation energies, the minimum of which is provided by Einstein's law stating that the energy (E) is equal toPlanck's constant(h) times the frequency of light $(v)$, or Planck's constant times the velocity of light(c) in a vacuum divided by its wavelength $(\lambda)$; that is,

$$
\mathrm{E}=\mathrm{hv}=\overline{\mathrm{h}}(1)
$$

In most cases, the excitation energies are normally higher than the energies needed to lift the electrons to a radioactive energy level, for instance, the luminescence produced by phosphor crystals in TV screens is excited by cathode ray electrons having energies of approximately 25,000 electron volts. Nevertheless, the color of the luminescent light is virtually independent of the energy of the exciting particles, mainly depending on the level of excited-state energy of the crystals.

The luminescence principle requires some excitation energy of different amounts depending on the color of the light. For instance, the excitation energy required for red light is 40 kilocalories, approximately 60 kilocalories 
for a yellow light, and approximately 80 kilocalories for violet light, all per mole of a substance. The excitation energy is however transferred to the electrons responsible for the luminescence, hence causing the electrons to jump from the ground-state energy level to a higher energy level. The electrons, positive ions, or photons of the luminous materials are initiated by mechanisms of excitation, by an impact that accelerates the particles, hence producing luminescence.

The electrons participating in the luminescence process are usually the outermost electrons of the exciting atoms and molecules. When gaseous molecules are excited, their luminescence spectra appear in broad bands, since not only electrons are lifted to higher energy levels but vibrational and rotational movements of the atoms are also entirely excited simultaneously. This is caused by the vibrational and rotational energies of the molecules which are 10-2 and 10-4 respectively. The electronic transition energies are represented by multiple of slightly different wavelengths that make up a band. Therefore, in molecules as well as atoms, the excited electrons are generally in the outermost molecular and atomic orbitals, hence causing the luminescence visible.

Industrial application of the luminous principle cut across fields such as intelligent lighting, car, and motor vehicle headlights, traffic lights, and displays such as televisions, billboards, and others and also decorative lighting which have taken the lead over the recent years. The LEDs have been of great use in the industrial field due to its low cost, high reliability, energy efficiency, and availability of peripheral/support systems and infrastructure. Additionally, the lasers have also been of great application in the industrial field, in areas such as communication, printing, display, data storage among others. This has been due to their high reliability, high quality of its spectrum, energy efficiency. Unfortunately, the market niche for LASERS can be at a slightly higher price but worth the task the lasers perform.

\section{The Difference between Light Emit- ting Diode (LED) Lamp and Light Ampli- fication by Stimulated Emission of Ra- diation (LASER).}

The laser and LED are the two common types of semiconductor light sources. The main difference between laser and LED lies in their operation principle, the junction area or the structure, concentration of charges, speed, transmitting distance, cost, and the type of light they produce. The list below describes the difference between laser and LED light sources.

Type of light emitted: The LED emits light comprising of different colors while a LASER produces light consisting of a single color. The LASER produces light of only a specific color because it contains only one wavelength, which is subject to the quantity of energy released when the excited electron falls to the lower orbit. The light produced by a LASER is also directional. On the other hand, the LED produces lights of different colors since it uses different materials that produce photons at varying wavelengths. The different wavelengths look like the light of various colors.

Working Principle: The LED works basically on the principle of electro-luminance, meaning that it gets emitted by utilizing electrons. The emitted light is rambled, that is, photons are in unsystematic phase among themselves. In addition, LASER operates on the principle of stimulated emission. LASER holds a coherent beam with a matching phase relative to the emitted photons. [4]

The output power: The lasers concentrate all their power to a small point, making them ideal for laser acupuncture. The output power is however high compared to the current above the threshold. On the other hand, the power emitted by LED is relatively lower and linearly proportional to the current driving it.

Speed, Transmitting Distance \& Cost: The LEDS transmits light in a standard short light source. Moreover, Lasers are however more powerful and operate at higher speeds than the LEDs. Lasers also transmit light to father distances and with fewer errors than the LEDs. In terms of cost, lasers are more expensive compared to LEDs.[5]The structure of the Junction: The junction area is another major difference between LED and LASERs. The Lasers have an extremely narrower area of the junction because the light is allowed to pass from an extremely small area in the LASER case. Besides, the junction area of LEDs is wider, thus allowing light to pass over a wider area.

The concentration of Charge Carriers: The concentration of charges refers to the concentration of holes and electrons. The concentration of charges in the laser is very high compared to that in LEDs. Therefore, this makes it possible for the laser to be used as a surgical instrument in the medical field among other fields because it possesses enough energy to even cut through objects.

\section{Conclusion}

From the above discussion, we can conclude that luminescence is a concept we use in our daily sight. The efficiency of luminescence emission is therefore subject to energy and a quantum basis. When all the exciting photon yields an emitted photon of the same energy, the efficiency of the luminescence is produced concerning the amount of input energy, as well as the number of quanta. However, the quantum efficiency of most luminescence is much lower than a hundred percent. LEDs and LASERS are so far the main types of luminescence in the application in day-to-day activities such as lighting purposes, in the health sector for diagnosis of internal body parts, for displays among other many applications across various fields. Therefore, efforts should be made by scientists to increase the excitation intensity, the density, and lifespan of the radioactive atoms and molecules to increase luminescence efficiency. Having known the various characteristics of luminescence such as excitation, emission of electrons and ions, the structure of the semiconductor materials, and other distinct working mechanisms, it is easier to develop further the luminescence principle through successful experimental setups. 


\section{Acknowledgement}

First of all, I would like to express my deepest gratitude to my tutor Professor Xie and the teaching assistants, who have provided me with valuable guidance in every stage of my thesis writing. Without Professor Xie's inspiring guidance and superior teaching level, I could not have finished my paper.

\section{References}

1. Adachi, C. (2014). Third-generation organic electroluminescence materials. Japanese Journal of Applied Physics, 53(6), 060101.

2. Lüssem, B., Riede, M., \& Leo, K. (2013). Doping of organic semiconductors. physical status solidi (a), 210(1), 9-43.

3. Nisoli, M. (2016). Semiconductor photonics. Principles and Applications. Società Editrice Esculapio.

4. Wierer Jr, J. J., Tsao, J. Y., \& Sizov, D. S. (2013). Comparison between blue lasers and light-emitting diodes for future solid-state lighting. Laser \& Photonics Reviews, 7(6), 963-993.

5. Heiskanen, V., \& Hamblin, M. R. (2018). Photobiomodulation: lasers vs. light-emitting diodes?. Photochemical \& Photobiological Sciences, 17(8), 1003-1017.

6. Arpad A. Bergh,P.J.Dean, Light-Emitting Diodes, Proceedings of the IEEE, Invited paper, 1972,60(2).

7. Penetration testing auditability. https://homepages.staff.os3.nl/ delaat/rp/20152016/p56/presentation.pdf 